\title{
Aula Inversa desde la virtualidad del Centro Metalmecánico para la Gestión de Tecnología y Buenas Prácticas formativas
}

\author{
Reverse Classroom from the virtuality of the Metalworking Center for Technology \\ Management and Good Training Practices
}

Iris A. Jiménez-Pitre ${ }^{1}$, Juan Felipe González-Molina² y Rubén Darío Cárdenas-Espinosa ${ }^{3}$

\section{(0) EDICIÓN: \#E-CIVTAC}

Recibido: 8/julio/2020

Aceptado: 20/agosto/2020

Publicado: 25/septiembre/2020

我 Colombia

${ }^{2}$ Colombia

${ }^{3}$ Colombia

\section{IIIIInstitución}

${ }^{1}$ Universidad de Caldas

${ }^{2}$ Universidad de Caldas

${ }^{3}$ Universidad de la Guajira

\section{Correo Eletrónico}

1iajimenez@uniguajira.edu.co 2jfgonzalezm@misena.edu.co 3Rdcardenas75@misena.edu.co

\section{ORCID}

1https://orcid.org/0000-0002-8109-7013 ${ }^{2}$ https://orcid.org/0000-0001-7829-2865 ${ }^{3}$ http://orcid.org/0000-0002-2417-844X

\section{Citar así: GCAPA / IEEE}

Jiménez-Pitre, I., González-Molina, J. \& Cárdenas-Espinosa, R. (2020). Aula Inversa desde la virtualidad del Centro Metalmecánico para la Gestión de Tecnología y Buenas Prácticas formativas. Revista Tecnológica-Educativa Docentes 2.0, 9(2), 59-70. https://doi.org/10.37843/rted.v9i2.145

I. Jiménez-Pitre, J. González-Molina y R. Cárdenas-Espinosa, "Aula Inversa desde la virtualidad del Centro Metalmecánico para la Gestión de Tecnología y Buenas Prácticas formativas", RTED, vol. 9, n. ${ }^{\circ} 2$, pp. 59-70, sep. 2020.

\section{Resumen}

Ante el confinamiento provocado por la Pandemia Covid-19, se forzó al cambio de lugar a fin de realizar actividades, al replantearse los deberes en clase y la lección en casa, apoyada por la tecnología, a través de ambientes virtuales de aprendizaje. El objetivo de este proyecto consistió en aplicar el Aula Inversa desde la virtualidad del Centro Metalmecánico en la Gestión de Tecnología con Buenas Prácticas formativas. La metodología corresponde a una investigación cualitativa con enfoque empírico analítico, carácter descriptivo de corte transversal, desarrollada en 4 fases Análisis, Diseño, Ejecución y Evaluación. De esta manera brindó una visión informal, que le permitió, tanto al instructor, como a sus aprendices, el acercarse más a la realidad, utilidad y aplicabilidad de los Ecosistemas Tecnológicas trabajados desde el Semillero de Investigación E-Innova CMM del Centro Metalmecánico, que han sido transferidos a la Universidad de Caldas, Centro de Comercio y Servicios SENA Regional Caldas, Universidad de la Guajira. El resultado obtenido fue, generar un modelo escalable, configurable con la tecnología, estrategias de uso, trabajo colaborativo, a partir de los Semilleros de Investigación E-Innova, Semilla GRICS, Grupos de Investigación GICEMET, GRICS, ReNuevaTe Ciencia, Tecnología e Innovación, BIEMARC con la Facultad de Ingenierías Universidad de Caldas y el semillero de investigación TECSIS. Se logró incrementar la actividad, aprendizaje significativo, desarrollo de proyectos tecnológicos por parte de los estudiantes e incentivar el uso de recursos educativos digitales por parte de los docentes.

Palabras clave: Aula Inversa, TIC, Entornos virtuales de aprendizaje, Investigación Aplicada, Gestión Tecnológica y Buenas Prácticas.

\section{Abstract}

Faced with the confinement caused by the Covid-19 Pandemic, they were forced to change places in order to carry out activities, by rethinking their homework in class and the lesson at home, supported by technology, through virtual learning environments. The objective of this project was to apply the Inverse Classroom from the virtuality of the Metalworking Center in the Management of Technology with Good Training Practices. The methodology corresponds to a qualitative research with an empirical analytical approach, descriptive character of cross-section, developed in 4 phases Analysis, Design, Execution and Evaluation. In this way, he provided an informal vision, which allowed both the instructor and his apprentices to get closer to the reality, utility and applicability of the Technological Ecosystems worked from the E-Innova CMM Research Seedbed of the Metalworking Center, which They have been transferred to the University of Caldas, SENA Regional Caldas Trade and Services Center, University of La Guajira. The result obtained was to generate a scalable model, configurable with technology, use strategies, collaborative work, from the E-Innova Research Seed, GRICS Seed, GICEMET Research Groups, GRICS, ReNuevaTe Ciencia, Tecnología e Innovación, BIEMARC with the Universidad de Caldas Faculty of Engineering and the TECSIS research hotbed. It was possible to increase activity, meaningful learning, development of technological projects by students and encourage the use of digital educational resources by teachers.

Keywords: Reverse Classroom, TIC, virtual learning environments, applied research, technological management, good practices. 
Aula Inversa desde la virtualidad del Centro Metalmecánico para la Gestión de Tecnología y Buenas Prácticas formativas.

\section{Introducción}

Debido al surgimiento del coronavirus 2019nCOV (COVID-19), que brotó finalizando el año 2019 en Wuhan, China, catalogado el 30 de enero de 2020 como Pandemia por la Organización Mundial de la Salud (OMS) (al extenderse en todos los países del mundo infectando una gran cantidad de personas), esta emergencia de salud pública preocupa a la comunidad internacional al causar manifestaciones respiratorias, digestivas como sistemáticas que afectan la salud humana (PérezThen, 2020). La mayoría de los países del planeta cerraron temporalmente sus instituciones educativas en un intento por contener su propagación ( $\mathrm{Lu}$, Stratton \& Tang, 2020) (Huang, Wang, Li, Ren, Zhao, Hu \& Cheng, 2020) (Liu \& Saif, 2020). Estos cierres a nivel nacional han afectado a más del $91 \%$ de la población estudiantil del mundo.

En otros países se implementaron cierres localizados que impactaron a millones de estudiantes adicionales. (Unesco, 2020). Botero (2020), en Colombia esta medida conlleva varios desafíos tan preocupantes como la llegada del virus, uno de ellos es el acceso a internet (principal), ya que, veinte millones de personas no tienen conexión, quienes sí la tienen, acceden solo, a través de su teléfono móvil, al exigir (Barrios, 2020) la sustitución de planta física por el ecosistema virtual forzando a que se incorporen e interactúen en comunidades virtuales estableciendo círculos comunes de acción, instituir en las redes sociales un ambiente para leer, escribir, construir, ubicar contenidos colaborativos de su autoría.

El confinamiento como medida de prevención, provocado por la Pandemia Covid-19, hizo que todas las organizaciones del planeta fueran forzadas al cambio de lugar de realización de sus actividades tradicionales presenciales haciéndolas de forma remota con distanciamiento social apoyados con tecnología, tal como ha sucedido en con el Centro Metalmecánico SENA Distrito Capital, Centro de Comercio y Servicios del SENA Regional Caldas, Universidad de la Guajira, Universidad de Caldas, quienes se vieron forzados a realizar teletrabajo desde casa debido a la cuarentena, esto, planteó que todos los instructores de formación presencial orientaran sus clases apoyadas en Tecnología. Una forma de afrontar este nuevo escenario garantizando la continuidad de los procesos de formación es el inversa o aula invertida como modalidad de aprendizaje semipresencial utiliza las estrategias virtuales, presenciales transfiriendo el proceso de enseñanza aprendizaje fuera del aula aprovechando las potencialidades de la web 2.0.

El Aula inversa inició a partir de dos experiencias dadas en Estados Unidos de América, la primera en una Escuela de Colorado 2006, como estrategia para disminuir la deserción, grabando los contenidos de clase a través del canal YouTube que se convirtió en un material de consulta libre ampliamente usado a nivel mundial (Bergamann \& Sams, 2014), la segunda formulada por Khan fundador de la academia Khan como refuerzo a sus primos menores en Matemáticas quienes no podían tener acceso presencial, optó por grabar videos en YouTube (Marin, 2015), lo cuales tuvieron un éxito inmediato (Khan, 2012), logrando que prefirieran los videos más que las clases presenciales,. En ambos casos se utilizaban los espacios de clase presencial al desarrollar las actividades prácticas y ejercicios que antes se dejaban en la casa, gracias a la programación de rutas temáticas con una evaluación guiada paso a paso (Marqués, 2016, p.13, Rincón \& Castilla, 2018).

En Colombia hay escasez de investigaciones sobre Aula Inversa, el primer antecedente es el informe de Paz, Serna, Ramírez, Valencia \& Reinoso, (2014) con la experiencia de aplicación de este modelo argumentando ventajas $y$ recomendaciones para su implementación efectiva. El cambio de lugar en la realización de actividades planteó la necesidad de cambiar disruptivamente lo que antes se realizaba en los ambientes de formación de la institución, por el trabajo en casa usando la Tecnología, a través del Campus Virtual, redes Sociales y herramientas de videoconferencia, cambiando el lugar y no el orden.

De esta manera se dio una visión informal que permitió tanto a instructor como a sus aprendices el acercarse más a la realidad, aprovechando esto, el desarrollo obtenido en los Ecosistemas Tecnológicos (García-Holgado \& García-Peñalvo, 2013a; 2013b; 2014; 2015; García-Holgado et al, 2015) elaborados desde el Semillero de Investigación E-Innova CMM del Centro Metalmecánico (Llorens, 2009; 2011; García-Peñalvo et al., 2015c; 2015d).

Desde el Centro Metalmecánico se hizo la transferencia tecnológica de lo desarrollado desde su Semillero y Grupo de Investigación GICEMET a los programas especiales de la Facultad de Ingenierías en 
Aula Inversa desde la virtualidad del Centro Metalmecánico para la Gestión de Tecnología y Buenas Prácticas formativas.
CONGRESO INTERNACIONAL VIRTUAL SOBRE LAS TECNOLOGÍAS DEL APRENDIZAJE Y DEL CONOCIMIENTO la Universidad de Caldas, el Centro de Comercio y Servicios SENA Regional Caldas, así mismo, a la Universidad de la Guajira.

La metodología utilizada, correspondió a una investigación cualitativa, a través del Modelo PACIE (Presencia, Alcance, Capacitación, Interacción, ELearning) con enfoque empírico analítico, carácter descriptivo de corte transversal, desarrollada en 4 fases Análisis, Diseño, Ejecución y Evaluación, resultado de la Investigación Doctoral Inclusión tecnológica educativa a través de Tecnologías de la Información y Comunicación (TIC) y el B-Learning.

Para el 2019, en el Centro Metalmecánico a través del Instructor Devia (2019) aplicó esta investigación, a través de formación complementaria presencial combinando el B-Learning, plataformas ELearning como NeoLMS, Blackboard, Moodle, Territorium aplicando políticas institucionales según lo planteado por Mujica-Sequera (2020), recursos educativos digitales del semillero de investigación a 240 Aprendices del Instituto Técnico Industrial Francisco José de Caldas, desde el Semillero de Investigación e Innovación E-InnovaCMM como eje articulador desde la virtualidad. Logrando a partir de ésta, se asesoró la creación de los Semilleros de Investigación en los programas Tecnológicos como RELEC (Tecnología en Electrónica), TECSIS (Tecnología en Sistemas Informáticos) en la Universidad de Caldas.

Se conformó una mesa de trabajo "Mesa 5_01. E-inclusión de tecnologías apropiadas para Interacción Social en Infancias - Juventudes" cuya participación fue en calidad de organizadores del evento III Bienal Internacional de Infancias y Juventudes que contó con 17 Ponencias evaluadas durante el tercer trimestre del 2018.

A partir del 2019 se inició con la Dinamización del Semillero de Investigación TECSIS en los Municipios del Eje Cafetero (Dorada, Manizales, Riosucio), brindando los fundamentos teóricos prácticos para el desarrollo de Diseños Digitales aplicables a proyectos de investigación aplicada, desarrollo tecnológico e innovación, mediante soluciones tecnológicas a los problemas del contexto local.

\section{Objetivo General}

Aplicar el Aula Inversa desde la virtualidad del Centro Metalmecánico para la Gestión de Tecnología y Buenas Prácticas formativas en los programas especiales de Ingeniería Informática, Tecnología en Sistemas Informáticos de la Universidad de Caldas.

\section{Objetivos Específicos}

1. Analizar la situación a mejorar en 3 asignaturas de los programas especiales en Ingeniería Informática, Tecnología en Sistemas Informáticos de la Universidad de Caldas que permitan realizar la determinación de indicadores medibles de mejora.

2. Diseñar estrategias didácticas activas que permitan la aplicación del Aula Inversa desde la virtualidad del Centro Metalmecánico para la Gestión de Tecnología y Buenas Prácticas formativas en los programas seleccionados.

3. Ejecutar el diseño propuesto en las asignaturas seleccionadas conforme a las políticas institucionales de la Facultad de Ingeniería de la Universidad de Caldas.

4. Evaluar los resultados obtenidos por los estudiantes a partir de los recursos educativos digitales aplicados por los docentes.

A continuación, está el Marco Teórico, la metodología, resultados, discusión, seguido de las conclusiones, por último, las referencias bibliográficas.

\section{Marco Teórico}

Debido a la Pandemia Generada por el COVIT-19, son grandes retos que se vienen en la humanidad en los próximos años, entre ellos se encuentra la educación; Por otra parte, la Comisión Europea define el E-Learning como el uso Tecnologías de Internet, multimedia, mejorar la calidad de enseñanza a través del acceso a los recursos de los servicios educativos, así como permitir la evaluación remota, el intercambio, colaboración entre estudiantes con profesores (Porras, Castilla, \& Rivera, 2017).

En el caso de las Instituciones educativas es relevante el uso de la gestión tecnológica en las buenas prácticas en la formación, las cuales se pueden desarrollar a través del benchmarking 
Aula Inversa desde la virtualidad del Centro Metalmecánico para la Gestión de Tecnología y Buenas Prácticas formativas.
CONGRESO INTERNACIONAL VIRTUAL SOBRE LAS TECNOLOGÍAS DEL APRENDIZAJE Y DEL CONOCIMIENTO (proceso sistemático, continuo de evaluación de los productos, servicios, procedimientos de trabajo de de las empresas, las cuales se reconocen como representantes de las mejores prácticas, cuyo propósito es el mejoramiento organizacional).

La gestión tecnológica consiste en un conjunto de actividades, herramientas y técnicas que integran ciencia, tecnología, con los procesos de las organizaciones y su infraestructura para fortalecer, corregir debilidades e incrementar su competitividad en sus productos y servicios aprovechando su capacidad tecnológica (Thamhain, 2005, Fuentes, Ramirez, Vargas \& Carrillo, 2012) citado por (Fuentes, Prada, Vargas \& Caicedo, 2011), en el caso de los Semilleros de Investigación del Centro Metalmecánico en articulación con la Facultad de Ingeniería de la Universidad de Caldas, se han desarrollado proyectos como aplicar las TIC en educación, lo cual, ha contribuido a simular lo real en el campo virtual con la metodología de trabajo PACIE (Presencia, Alcance, Capacitación, Interacción y ELearning) (Cárdenas, Salazar \& Ruiz, 2018, p. 14) propuesta por Camacho (2009), con el modelo de aula inversa.

Un ambiente virtual de aprendizaje (AVA) facilita la interacción, guía y orientación del instructor con los aprendices en todo su proceso de aprendizaje (Domínguez, Rama \& Rodríguez, 2013), incorporando las (TIC) a los entornos virtuales de aprendizaje (EVA), el cual, constituye el mecanismo ideal utilizando el aula inversa como modelo donde se invierten los momentos y roles tradicionales de la educación, presentando los temas de clase disponibles desde su hogar, usando herramientas o plataformas multimedia que originalmente el instructor realizaba en clase, dejando al realizar en clase trabajo colaborativo, aprendizaje basado en problemas y realización de proyectos (Coufal, 2014; Lage, Platt y Treglia, 2000; Talbert, 2012).

Los elementos del Modelo de Aula Inversa se pueden consolidar en 3 según su uso así:

1. Modelo Simple, aquí se incorpora la lección en casa a través de videos; los deberes o tareas se realizan durante la clase resolviendo dudas y preguntas del video siendo esto una continuación de la clase (García \& Rodríguez, 2016).

2. Modelo de observación, en él, la lección en casa aparte de los videos incluye cuestionarios con información y foros de dudas e inquietudes; los deberes o tareas en clase se destinan en aclarar las dudas y formular preguntas del video como continuación de la clase (Jorge, 2016).

3. Modelo innovador, involucra a la lección en casa micro actividades a realizar sobre el video propuesto, empleo de los resultados de actividades de la sesión presencial en clase permiten reestructurar los cuestionarios propuestos y los foros de dudas e inquietudes; los deberes o tareas en clase se programan en el desarrollo de evidencias como recurso didáctico y continuación de la clase (Moya, 2017).

\section{Metodología}

Los Ecosistemas Tecnológicos mediados por (TIC) para la Investigación formativa en contexto, gestionan el conocimiento generado en los proyectos de aula en los programas Tecnológicos Universitarios, aplicando el B-Learning a través del Modelo PACIE (Presencia, Alcance, Capacitación, Interacción, E-Learning) (Cárdenas, Salazar, \& Ruiz, 2018, p. 14) a través de los Semilleros de Investigación E-InnovaCMM del Centro Metalmecánico Distrito Capital, Semilla GRICS del Centro de Comercio y Servicios del SENA Regional Caldas, TECSIS Universidad de Caldas.

La metodología empleada corresponde a una investigación cualitativa con enfoque empírico analítico, carácter descriptivo, corte transversal, resultado de la Investigación Doctoral Inclusión tecnológica educativa a través del B-Learning (TIC). Se realizó en 4 fases Análisis, diseño, ejecución y evaluación.

\section{Fases del Proyecto}

1. Análisis: Se realizó la identificación de la situación a mejorar planteando indicadores medibles de mejora a partir de la experiencia del Semillero de Investigación EInnovaCMM, además se identificó la situación a mejorar en 3 asignaturas de los programas especiales en Ingeniería Informática, Tecnología en Sistemas Informáticos de la Universidad de Caldas que permitan realizar la determinación de indicadores medibles de mejora.

2. Diseño: Se estructuró las estrategias 
didácticas activas que permitan la aplicación del Modelo Aula Inversa desde la virtualidad del Centro Metalmecánico para la Gestión de Tecnología y Buenas Prácticas formativas.

3. Ejecución: En esta fase se Implementó el Modelo diseñado utilizando el Modelo PACIE (Planeación, Alcance, Capacitación, Interacción y E-Learning), en las asignaturas seleccionadas conforme a las políticas institucionales de la Facultad de Ingeniería de la Universidad de Caldas.

4. Evaluación: Consiste en la Validación del Modelo Implementado en procesos de formación en Campo que tenían una formación Tradicional presencial, a partir de los resultados obtenidos por los estudiantes a partir de los recursos educativos digitales aplicados por los docentes.

\section{Resultados}

El resultado obtenido fue, generar un modelo escalable, configurable con la tecnología y estrategia de uso. Esta experiencia ha permitido aplicar el aula inversa en formación complementaria virtual, a través de, cursos como Controladores Lógicos Programables (PLC), Aplicación de los PLC en la Automatización de Procesos Industriales, Los PLC en los Sistemas SCADA, Introducción a los Sistemas de Automatización, Servicios de Automatización que se dan en el Centro Metalmecánico del SENA Distrito Capital, como en asignaturas de los programas especiales de Ingeniería Informática, Tecnología en Sistemas Informáticos de la Universidad de Caldas, a través del trabajo colaborativo entre el Semillero de Investigación EInnovaCMM, el Grupo de Investigación GICEMET del Centro Metalmecánico, ReNuevaTe Ciencia, Tecnología e Innovación, semillero de Investigación TECSIS de la Universidad de Caldas.

A continuación, se presentan las experiencias o anécdotas durante el confinamiento a través a la utilización de esas herramientas tecnológicas para la virtualidad durante el proceso educativo:

\section{Acondicionamiento de sesiones Presenciales a una forma de virtualización apoyada en Tecnología para Ingeniería Informática.}

Materia orientada: Gestión Tecnológica y Buenas Prácticas. Listado de los estudiantes que asisten a las clases virtuales a través del siguiente formulario en línea https://docs.google.com/spreadsheets/d/1ErkWe4s mcZ_QzQOgFFc9sLAxsF1lj_bkxKDz2o7tVkk/edit ?usp=sharing

$\checkmark$ Logros obtenidos: cada uno de los estudiantes, equipos de trabajo realizaron a cabalidad las correcciones a las observaciones presentadas en el Tema de Clase "Modelo COBIT" a una Empresa seleccionada por cada equipo de estudiantes durante el trabajo colaborativo desarrollado en clase, quienes hicieron en casa las correcciones pertinentes, validadas, debidamente socializadas en sesión Sincrónica de clase empleando la herramienta de videoconferencia Zoom. Se realizó el diseño de 6 propuestas de implementación del Modelo COBIT5 aplicable a las Empresas de la Ciudad de Manizales: Instituto Oftalmológico de Caldas, Becall Group, Panadería la Victoria, Prometálicos, COOPORECAL, La Suiza, a partir de las buenas prácticas identificadas en dos competidores identificados de su segmento de mercado a través del Benchmarking Tecnológico, utilizando instrumentos como Benchmarking, la Guía metodológica (COBIT5) Objetivos de Control para las Tecnologías de la Información Relacionadas, (ITIL) Infraestructura de Tecnologías de Información, la norma ISO27000 sobre seguridad informática, los cuales se documentaron a través de un artículo científico presentado en el XII Encuentro Departamental de Semilleros de Investigación de la Red Regional de Semilleros de Investigación para el Eje Cafetero \& Norte del Valle (RREDSI).

$\checkmark$ Inconvenientes: No manifiestan ninguno, han considerado acertadas la estrategia de trabajo a través del modelo de Aula Inversa con los videos y cuestionarios desarrollados por el docente, con la aplicación de técnica didáctica activa de Estudio de Caso y Resolución de Problemas en contextos reales. 
$\checkmark$ AVA: Uso campus Virtual Moodle https://cvirtual.campusvirtualudecaldas.edu.c o/moodle/course/view.php?id=1736

$\checkmark$ EVA: Se utilizó la red Social WhatsApp, alojando a través de un Grupo creado para la clase las evidencias de lo trabajado, con enlaces a recursos educativos digitales web 2.0 publicados en ISSUU, YouTube, Drive, Moodle.

$\checkmark$ Recursos: a continuación, se presentan los siguientes:

- Grupo WhatsApp para comunicación asignatura Gestión Tecnológica y Buenas Prácticas, https://chat.whatsapp.com/JnBDjcPw umfIR0kIKNdps 7.

- YouTube: https://youtu.be/k7Zu7gFn9Vk

COBIT5 modelo de implementación, https://youtu.be/1EuBA1q_nYA

Infraestructura de Tecnologías de Información ITIL, https://youtu.be/0bk_yirG8EA ISO 27000 - ISO 27001 Seguridad Informática.

- Correo electrónico ruben.cardenas@ucaldas.edu.co

- Videoconferencia Salas de Zoom https://zoom.us/j/235776973?pwd=R XFHdVRwdUJXQ2hxcldLNWwrMz dE https://zoom.us/j/99176701950

- Podcast SoundCloud Aquí se grabó audios cortos con los aspectos más relevantes para tener en cuenta Gestión Tecnológica y Buenas Prácticas https://soundcloud.com/ruben-dariocardenas-espinosa/2020-04-

20_112126aspectos-a

- ISSUU:

https://ISSUU.com/rubendariocarden asespinosa/docs/infraestructura_de_t ecnolog_as_de_informaci_n_iti Infraestructura de Tecnologías de Información ITIL

https://ISSUU.com/rubendariocarden asespinosa/docs/iso_27000__27001 _seguridad_inform_tica_rdce ISO 27000 - 27001 Seguridad Informática.

Inasistencias a clase: Hubo participación activa sin deserción.

\section{Acondicionamiento de sesiones Presenciales de Educación a Distancia en la Tecnología para Tecnología en Sistemas Informáticos}

Materia orientada: Coordinación de Servicios TI. Listado de los estudiantes que asisten a las clases virtuales a través del siguiente formulario en línea https://docs.google.com/spreadsheets/d/1OZ0Beju XeyjJSw1_5fJP8XJaJMo9LNbaHN76SRzhYHA/ edit?usp=sharing

Logros obtenidos: cada uno de los estudiantes, equipos de trabajo realizaron a cabalidad las correcciones a las observaciones dadas en para implementar el Tema de Clase "Instalación de Servicios TI a través de Linux Server, Windows Server" a una Empresa seleccionada por cada equipo de estudiantes durante el trabajo colaborativo desarrollado en clase, quienes hicieron en casa las correcciones pertinentes, validadas, debidamente socializadas en sesión Sincrónica de clase empleando la herramienta de videoconferencia Zoom. Se desarrollaron los procesos Help Desk (mesa de ayuda), coordinación de Servicios de Tecnologías de Información TI tomando como escenario de prueba las salas de cómputo de la Universidad de Caldas, empleando Software Libre para la administración de Incidentes incorporado como servicio adicional.

$\checkmark$ Inconvenientes: No manifiestan ninguno, han considerado acertadas la estrategia de trabajo a través del modelo de Aula Inversa con los videos y cuestionarios desarrollados por el docente, con la aplicación de técnica didáctica activa de Estudio de Caso y Resolución de Problemas en contextos reales.

$\checkmark$ AVA: Uso campus Virtual Moodle https://cvirtual.campusvirtualudecaldas.ed u.co/moodle/course/view.php?id=1589

$\checkmark$ EVA: Se ha utilizó la red Social WhatsApp, alojando a través de un Grupo 
La clase las evidencias de lo trabajado, con enlaces a recursos educativos digitales web 2.0 publicados en ISSUU, YouTube, Drive, Moodle.

$\checkmark$ Recursos: a continuación, se presentan los siguientes:

○ Grupo WhatsApp para comunicación asignatura Coordinación de Servicios TI, destinado para brindar apoyo en coordinación de servicios TI tanto de forma síncrona como asíncrona desde el 11 febrero cuando iniciaron las clases.

- YouTube:

https://youtu.be/PTquke3mTGM

Impresoras: Matricial, Inyección de Tinta y Láser.

- Recursos web

2.0 https://blog.comparasoftware.co

$\mathrm{m} / \mathrm{mesa}$-de-ayuda-usos-y-

aplicaciones/; https://www.comparaso ftware.com/mesa-de-ayuda/, Se dejó libertad de las versiones disponibles de evaluación enlace https://www.microsoft.com/es$x l / e v a l c e n t e r / e v a l u a t e-w i n d o w s-$ server-2016 y utilizar Azure, ( ISO o Virtual Labs) las máquinas virtuales para dejar el servidor listo para la configuración de servicios de red, con una máquina virtual sobre el sistema operativo del equipo si instaló Windows en la máquina virtual instala Linux y viceversa.

- Correo electrónico ruben.cardenas@ucaldas. edu.co

- Videoconferencia Salas de Zoom https://zoom.us/j/235776973?pwd=R XFHdVRwdUJXQ2hxcldLNWwrMz dEQT09 Tema Instalación de un sistema operativo Server (Windows 2012 o 2016 o 2018 Windows server o Linux Ububthu 12, 14 o la más reciente) al convertir todas las configuraciones básicas (drivers, red, entre otros servicios).

Inasistencias a clase: Hubo participación activa sin deserción.
Materia orientada: Gestión de Proyectos

TI. Listado de los estudiantes que asisten a las clases virtuales a través del siguiente formulario en línea

https://docs.google.com/spreadsheets/d/1UNadjO W2TgWslorg5gJCPqQ6MYhEGAltjENhyFn4Rd A/edit?usp=sharing

$\checkmark$ Logros obtenidos: Se estructuraron 7 proyectos de gestión de TI, cada uno de los estudiantes, equipos de trabajo realizaron a cabalidad las correcciones a las observaciones dadas en para implementar los Temas de Clase "Mapa de empatía, Escenarios de uso, Diseño de encuestas a través de formularios web, Estructuración de un proyecto TI, Diseño de Póster, Escritura de Artículo Científico, Modelo CANVAS, Registro derechos de Autor, Divulgación Científica". Se ha realizado retroalimentación personalizado, todos los que han cumplido con las observaciones, entregas han realizado las correcciones de forma completa con la calidad solicitada. Solo 2 estudiantes se atrasaron por causas de fuerza mayor.

$\checkmark$ Inconvenientes: Inconformidades de la metodología de trabajo por parte de los estudiantes atrasados por considerar que tuvieron sobrecarga al dejarse alcanzar con las actividades a realizar en clase por inasistencia y no destinar tiempo en casa para estudiar los videos, cuestionarios y foros dispuestos en el desarrollo de la asignatura, además manifestaron saturación de información enviaba por el Chat del Grupo, por este motivo se acordó no enviar comunicados en días diferentes al día de clase programada, recordando la sesión; se aclaró.

$\checkmark$ AVA: Uso campus Virtual Moodle Aula Semillero de Investigación TECSIS.

$\checkmark$ EVA: Se ha utilizó la red Social WhatsApp, alojando a través de un Grupo creado para la clase las evidencias de lo trabajado, con enlaces a recursos educativos digitales web 2.0 publicados en ISSUU, YouTube, Drive, Moodle.

$\checkmark$ Recursos: a continuación, se presentan los siguientes: 
Aula Inversa desde la virtualidad del Centro Metalmecánico para la Gestión de Tecnología y Buenas Prácticas formativas.
CONGRESO INTERNACIONAL VIRTUAL SOBRE LAS TECNOLOGÍAS DEL APRENDIZAJE Y DEL CONOCIMIENTO
- Grupo WhatsApp para comunicación asignatura Gestión

- Proyectos TI, destinado para brindar apoyo en coordinación de servicios TI tanto de forma síncrona como asíncrona desde el 11 febrero cuando iniciaron las clases.

- YouTube:

https://www.youtube.com/watch?v=gSX onUg95AA\&t=30s Metodologías de investigación de los semilleros EInnovaCMM y TECSIS desde la virtualidad,

https://www.youtube.com/watch?v=TKV NL88bF7s\&t=283s Estado del Arte, Mapa de Empatía y Escenarios de Uso, https://www.youtube.com/watch?v=9hfe $\mathrm{kSsS1Ac \& t}=1690$ s Seminario Escritura de Artículos Científicos para Biólogos Universidad de la Guajira, https://www.youtube.com/watch?v=J2tL 74j1PnU OVA Estado del Arte de un Proyecto, https://www.youtube.com/watch?v=hQv OYAFPjr8\& $\mathrm{t}=275 \mathrm{~s}$ Aspectos calve para la escritura de un artículo científico.

- Recursos web 2.0 http://edublogproyectodegrado.blogspot. com/ Edublog Proyecto de Grado.

- Correo

electrónicoruben.cardenas@ucaldas. edu.co

- Videoconferencia Google Meet https://meet.google.com/hmc-okvqxso Zoom https://zoom.us/j/96901590855

$\checkmark$ Inasistencias a clase: Hubo participación activa sin deserción. Solo dos estudiantes no asistieron a 3 clases sincrónicas, una estudiante no pudo conectarse a una sesión, pero dejó representación en un compañero y al día siguiente se le aclararon las inquietudes.

\section{Sistematización de experiencias en la Escuela Nacional de Instructores del SENA}

Por parte de la Escuela Nacional de Instructores se participó en la convocatoria Instructores Inspiradores en época de cuarentena, presentando las estrategias como la desarrollada en esta investigación, demostrando el compromiso y entrega con la Formación Profesional Integral. La Figura 1 muestra los resultados obtenidos, en la cual, se pudo dar a conocer cómo día a día se inspiran a los aprendices a seguir adelante, a no dejarse vencer, creando estrategias didácticas activas, continuando con la formación desde casa.

\section{Figura 1.}

Reconocimiento Estrategia Aula Inversa por parte de la Escuela Nacional de Instructores ENI SENA.

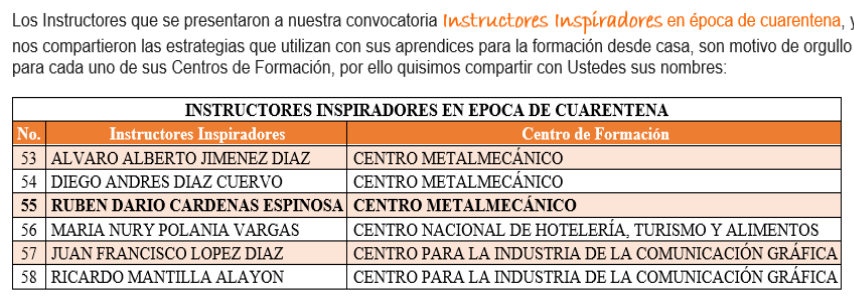
CENTRO PARA LA INDUSTRIA DE LA COMUNICACION GRAFICA

Estimados Instructores, muchas gracias por su compromiso, Ustedes son quienes hacen grande nuestra Institución. Felicitaciones.

Con gusto comparto el video que se publicó a través de comunicaciones Regional, el pasado 24 de junio, donde el Director Regional Distrito Capital, felicita muy especialmente a quienes participaron de esta convocatoria y podemos
identificar el rostro de varios de nuestros Instructores Inspiradores.

Desde la ENI estamos trabajando para crear espacios donde los Instructores Inspiradores que quieran compartir sus estrategias, lo hagan para que otros Instructores las conozcan. Estaremos invitándolos a participar de estos espacios, cuanto sean definidos.

Muchas gracias por su participación y les envío un cordial saludo.

Sandra Julissa Bustos Vidarte

Profesional

Nota. Resultados obtenidos, elaboración propia.

\section{Discusión}

En opinión de los expertos, la tecnología es una de las herramientas clave para que la imposibilidad de desplazarse a los centros de estudio no afecte a la educación de los alumnos, actualmente, el COVID-19 ha alterado de forma abrupta y acelerada la educación tradicional que debió realizarse en línea sin preparación o tiempo de transición por parte de todos los niveles académicos, como una solución factible. La educación en línea como sistema alternativo o complementario al presencial ante situaciones complicadas no es una novedad.

Aunque hay experiencias de cambio en los procesos educativos presencial a virtual como la Universidad de la Columbia Británica (Canadá) debido a los problemas de tráfico de Vancouver, la Universidad Johns Hopkins (Estados Unidos) con simulaciones multimedia, como alternativa de solución a la realización de autopsias formativas por la escasez de cuerpos (Sangrà, 2020), ilustran casos 
donde se pudo planificar, a partir de procesos controlados la migración de sistema educativo, pero cuando es un cambio abrupto como el que se generó por la pandemia, los autores consideramos que ha desnudado la realidad del sistema educativo actual, al considerarlo limitado al no garantizar la pertinencia, calidad, equidad que no puede seguir siendo orientada con trabajos mecánicos, rutinarios y repetitivos, que no enseñan a leer, pensar y convivir a nuestras próximas generaciones.

La realización de videos en el modelo de aula inversa no implica cambiar la formación presencial, constituye un caso del uso de tecnologías mejorando la formación, sin necesidad de cambiar la forma habitual de clase, se considera relevante, tener en cuenta la duración de estos, es un facilitador en la toma de apuntes siguiendo la explicación del tema, con la posibilidad de pararlo o repetir las veces que considere pertinentes desde la comodidad de su hogar. Esta investigación, permitió identificar que los videos con duración mayor de 10 minutos sin cuestionarios o actividades complementarias de apoyo no son vistos en su totalidad por los estudiantes, puesto que algunas veces se saltan partes, solo se enfocan en los elementos que les interesa así sean videos de corta duración.

Para el caso de los videos que se desarrollen bajo el modelo Aula Inversa coincidimos en las siguientes características en función de las necesidades comunes de los instructores así:

b. Calidad del video, en este aspecto es importante tener en cuenta su dependencia de los medios técnicos y profesionales requeridos en la grabación de este. Aquí juega un papel importante la personalidad del docente y sus características individuales, ya que, para algunos, si la grabación se hace en un estudio se puede divulgar, sino no lo harían nunca. Esto nos permite reflexionar sobre los siguientes interrogantes ¿qué implica cada tipo de video?, ¿qué calidad elegir? ¿en qué situaciones utilizarlos?

c. Duración del video, como se presentó al inicio de la discusión cuando se trabaja educación virtual o a distancia se recomiendo que la duración no sea mayor de 10 minutos, en el caso particular de los autores se unifico como criterio el tiempo máximo de 5 minutos si se va a enviar a través de una red social. Esto nos permite reflexionar acerca de las siguientes preguntas ies viable grabar toda una lección magistral en el método Aula Inversa? ¿qué duración es recomendable que tengan los videos? ¿por qué?

a. Interacción, esta característica permite desarrollar distintas formas en las que el instructor interactúa con los contenidos del video: de tipo tablero, tipo presentación y práctica.

Esta investigación nos permitió reflexionar sobre ¿Cuáles serían las habilidades adquiridas por parte de los estudiantes de la formación presencial del SENA, Universidad de Caldas o cualquier otra institución educativa, ¿ante la transición del ecosistema digital durante la pandemia del COVID19? A partir de esta pregunta, se plantearon 2 temas con el propósito de colocar en un contexto general a los participantes: El primer tema se relaciona con el impacto del Covid-19 en sus vidas. El segundo comprendió la percepción sobre la trasformación de las comunicaciones a nivel mundial en tiempos de pandemia.

Se pudo interpretar de forma resumida, a partir de la experiencia realizada, previa consulta a cada uno de los participantes de este proceso que, han desarrollado habilidades digitales para el aprendizaje, logrando una adaptación al cambio, aunque estuvieran familiarizados previamente con algunas de ellas, la abrupta transición al ecosistema digital, los obligó a cambiar de mentalidad (forma de pensar), luchar contra los sentimientos generados al inicio de ésta, lo cual se facilitó, gracias al apoyo docente logrando un aprendizaje significativo.

Un aspecto a mejorar en el éxito del modelo aula inversa es el técnico, hay factores exógenos como velocidad, características de equipos, conectividad, competencias de los docentes y alfabetización digital de los estudiantes, los cuales son determinantes en la implementación del desarrollo práctico de laboratorios con acceso remoto o mediante simuladores virtuales, cuyo medio de interacción en tiempo real son las clases en línea a través de Google Meet (herramienta de Google) por sus prestaciones, combinado a otras herramientas tecnológicas disponibles (Territorium, Moodle, YouTube, WhatsApp) en las cuales ejecutan los procesos de aprendizaje, acceso a contenidos, 
sesiones en línea, envío de las diferentes actividades a desarrollar.

\section{Conclusiones}

El desarrollo de este proyecto bajo la metodología de aula inversa en las asignaturas:

1. Gestión Tecnológica y Buenas Prácticas permitió el desarrollo de 6 propuestas constituyeron una línea base para aplicar en diferentes empresas que requieren estar a la vanguardia de las tendencias de los procesos tecnológicos de innovación del Siglo XXI;

2. Coordinación de Servicios TI brindó una guía en la administración, configuración adecuada de la infraestructura en servicios de red necesarios dentro de las organizaciones con el fin brindar dichos servicios a los diferentes usuarios, al tener en cuenta que hoy en día cualquier organización cuenta con por lo menos un servidor;

3. Gestión de Proyectos TI permitió la formulación estructurada de 7 proyectos con aplicaciones TI, donde cada uno ubicó en un Mapa de Empatía el punto de vista del cliente, muestra de forma creativa a través de un Escenarios de Uso el problema, su solución con la aplicación, diseño de una Encuesta con su respectiva aplicación, su posterior presentación en un póster digital con su Modelo CANVAS, registro de software propuesto en la página derechos de autor.

La interacción en tiempo real se destacó por la herramienta de videoconferencia Meet por su estabilidad en la conexión, acceso de cualquier usuario, grabación automática en la nube, y la facilidad de compartirla de forma automática el chat y video de clase.

Un caso exitoso de combinación de herramientas es apoyarnos en WhatsApp por su facilidad, cobertura, accesibilidad desde los dispositivos móviles, planes de los proveedores de red telefónica móvil que permiten su acceso gratuito garantizando la comunicación en tiempo real con los estudiantes, el acceso a los recursos educativos digitales bajo el modelo de aula inversa.
Los participantes evidenciaron el desarrollo de habilidades técnicas no solo desde los temas de cada asignatura, sino en su adaptación al cambio, forzados en el aprendizaje obligado de hardware y software, motivados por la abrupta transición al ecosistema digital en la pandemia del COVID-19, que los obligó a cambiar de mentalidad para luchar contra los sentimientos generados al inicio de ésta, cumpliendo no solo los objetivos trazados en cada proceso.

Gracias al modelo de aula inversa, se facilitó, la mediación del docente para lograr un aprendizaje significativo, en el cual, a pesar de las limitaciones técnicas en equipos, ancho de banda, conectividad a internet, se estructuraron laboratorios virtuales por medio de simuladores cumpliendo con los resultados de aprendizaje de cada asignatura.

\section{Reconocimiento}

Este proceso investigativo se ha logrado gracias al apoyo Incondicional del Subdirector del Centro Metalmecánico Dr. Jairo Iván Marín Masmela, Coordinadora Misional Dra Claudia María Martínez Zuluaga, equipo de trabajo de los grupos de Investigación GICEMET, ReNuevaTe Ciencia Tecnología e Innovación, SENNOVA, Dra. María Helena Mejía Salazar Decana de la Facultad de Ingenierías de la Universidad de Caldas, Dr. Fabio Andrés López Salazar Director de los programas especiales de Ingeniería Informática y Tecnología en Sistemas Informáticos de la Universidad de Caladas así como los aprendices que han participado en todos los procesos de formación de ambas instituciones.

\section{Referencias}

Barrios, A. (2020). COVID-19 abre agenda a la virtualidad de la educación colombiana. Recuperado de: https://www.pulzo.com/opinion/educacion-virtual-tiemposcoronavirus-PP874848

Bergmann, J. \& Sams, A. (2014) Flipped Learning: Gateway to Estudent Engagement. International Society for Technology in Education. Kindle Vertion.

Camacho, P. (2009). Metodología PACIE. Recuperado de: http://vgcorp.net/pedro/?page_id=20

Cárdenas, R. López, M. \& Agudelo, J. (2015). Blearning en los proyectos de innovación y desarrollo tecnológico del semillero Biometrónica del SENA Regional Caldas. CORDESEC. 
Cárdenas, R. (2017). Inclusión tecnológica educativa a través del BLearning y las tecnologías de información y comunicación (TIC). En Editorial Corporación Centro Internacional de Marketing Territorial para la educación y el desarrollo. Memorias CIMTED Séptima Edición COINCOM 2017 VI Congreso Internacional sobre Competencias Laborales COINCOM 2017. (801 - 818). Cartagena. Editorial Corporación Centro Internacional de Marketing Territorial para la educación y el desarrollo.

Cárdenas, R., Ruiz, J. \& Ruiz, C. (2018). Aprendizaje colaborativo NTIC de ciencia, tecnología e innovación desde los semilleros de investigación en programas tecnológicos. Tecnología $e$ Innovación + Ciencia e Investigación en América Latina, (10 25). Recuperado de: http://memoriascimted.com/wpcontent/uploads/2017/01/Tecnolog\%C3\%ADa-einnovaci\%C3\%B3n-libro-citici2018.pdf.

Coufal, K. (2014). Flipped learning instructional model: perceptions of video delivery to support engagement in eighth grade math. (Tesis doctoral). ProQuest, UMI Dissertations Publishing (UMI3634205)

Espinosa, R. (2015). Blearning en la formación del SENA. Revista Rutas de formación: Prácticas y Experiencias, (1), (44-51).

Espinosa, R. (2018). Tecnologías de Información y comunicación desde la virtualidad para la formación en investigación aplicada e innovación "caso semilleros de investigación en los programas tecnológicos Universidad de Caldas". Revista Hamut' ay, 5(1), (105-117).

Espinosa, R. \& Caicedo, L. (2018). Las NTIC en la investigación formativa desde la virtualidad, caso Semillero de Investigación e Innovación E-InnovaCMM del Centro Metalmecánico. Revista Rutas de formación: Prácticas y Experiencias, (7), 100-108.

Fuentes, M., Prada, D., Vargas, A., \& Caicedo, G. (2011). Gestión tecnológica: conceptos y casos de aplicación. Revista GTI, 10(26), 43-54.

Fuentes, M., Ramirez, P., Vargas, A. \& Carrillo, G. (2012). Gestión tecnológica: conceptos y casos de aplicación. Gerencia Tecnológica Informática, 10(26).

García-Holgado, A., \& García-Peñalvo, F. (2013). The evolution of the technological ecosystems: An architectural proposal to enhancing learning processes. In F. J.

García-Peñalvo, F. (2013), Proceedings of the First International Conference on Technological Ecosystems for Enhancing Multiculturality (TEEM'13). ACM.

García-Holgado, A., \& García-Peñalvo, F. J. (2016). Architectural pattern to improve the definition and implementation of eLearning ecosystems. Science of Computer Programming, 129, 20-34. https://doi.org/10.1016/j.scico.2016.03.010

García-Peñalvo, F. (2008). Docencia. In J. Laviña Orueta \& L. Mengual Pavón (Eds.), Libro Blanco de la Universidad Digital 2010 (p. 29-61). Ariel.

García-Peñalvo, F. (2015). Inteligencia Institucional para la Mejora de los Procesos de Enseñanza-Aprendizaje. Recuperado de: http://repositorio.grial.eu/handle/grial/406
García-Peñalvo, F. (2016a). Ecosistemas de Aprendizaje Adaptativos. Recuperado de: https://goo.gl/RCntka

García-Peñalvo, F. (2016b). ¿Son conscientes las universidades de los cambios que se están produciendo en la Educación Superior? Education in the Knowledge Society, 17(4), 7-13. doi:10.14201/eks2016174713

García-Peñalvo, F. (2016c). Technological Ecosystems. IEEE Revista Iberoamericana de Tecnologías del Aprendizaje, 11(1), 31-32. doi:10.1109/RITA.2016.2518458

García-Peñalvo, F. (2017a). Ecosistemas Tecnológicos: Innovando en la Educación Abierta. Recuperado de: https://goo.gl/zRma4d

García-Peñalvo, F. (2017b). El (des)gobierno de las tecnologías de la información en las universidades. Recuperado de: https://goo.gl/xmQVZD

García-Peñalvo, F., García de Figuerola, C., \& Merlo-Vega, J. A (2010a). Open knowledge management in higher education. Online Information Review, 34(4), 517-519.

García-Peñalvo, F., García de Figuerola, C., \& Merlo-Vega, J. A. (2010b). Open knowledge: Challenges and facts. Online $\begin{array}{lll}\text { Information } & \text { Review, } & \text { 34(4), }\end{array}$ doi:10.1108/14684521011072963

García-Peñalvo, F., \& García-Holgado, A. (Eds.). (2017). Open Source Solutions for Knowledge Management and Technological Ecosystems. IGI Global.

García, R., \& Rodríguez, M. (2016). El aula invertida (flipped classroom) en Educación Primaria. Recuperado de: https://www. researchgate.

net/publication/305474488_El_aula_invertida_flipped_classroo m_en_Educacion_Primaria.

Huang, C., Wang, Y., Li, X., Ren, L., Zhao, J., Hu, Y., ... \& Cheng, Z. (2020). Clinical features of patients infected with 2019 novel coronavirus in Wuhan, The Lancet, 395(10223), 497-506. https://doi.org/10.1016/S0140-6736(20)30183-5.

Jorge, A. (2016). El desarrollo de la interacción oral en la clase de E/LE (A2. 1) (Doctoral dissertation).

Khan, S. (2011). Let's use video to reinvetn education [video]. YouTube https://www.youtube.com/watch?v=nTFEUsudhfs

Lage, M., Platt, G., y Treglia, M. (2000). Inverting the classroom: A gateway to creating an inclusive learning environment. The Journal of Economic Education, 31(1), 30-43

Liu, S., \& Saif, L. (2020). Emerging viruses without borders: The Wuhan coronavirus https://doi.org/10.3390/v12020130.

Lu H, Stratton CW \& Tang YW. (2020). Outbreak of Pneumonia of Unknown Etiology in Wuhan China: The Mystery and the Miracle. J Med Virol. https://doi.org/10.1002/jmv.25678.

Llorens, F. (2009). La tecnología como motor de la innovación educativa. Estrategia y política institucional de la Universidad de Alicante. Arbor, 185(Extra), 21-32.

Marin, M. (2015). Un profesor con más de 26 millones de alumnos. Recuperado de: https://www.pagina12.com.ar/diario/sociedad/3283048-2015-10-04.html 
Aula Inversa desde la virtualidad del Centro Metalmecánico para la Gestión de Tecnología y Buenas Prácticas formativas.

Marqués, M. (2016). Qué hay detrás de la clase al revés. ReVisión. Revista de Investigación en docencia universitaria de la informática. 9(13) 11-18. Recuperado de http://www.aenui.net/ojs/index.php?journal=revision\&pa ge $=$ article\&op $=$ view $\& p$

Moya, J. (Ed.). (2017). La docencia universitaria mediante el enfoque del aula invertida. Ediciones Octaedro.

Mujica-Sequera, R. (2020). E-Learning como estrategia pedagógica en la educación superior. Revista TecnológicaEducativa Docentes 2.0, 9(1), 37-41. https://doi.org/10.37843/rted.v9i1.103

Paz, A., Serna, A., Ramírez, M., Valencia, T., \& Reinoso, J. (2014). Hacia la Perspectiva de Aula Invertida (Flipped Classroom) en la Pontificia Universidad Javeriana desde una tipología de uso educativo del Sistema Lecture Capture (S.L.C). En N. Duque (Presidencia), LACLO 2014: Novena Conferencia Latinoamericana de Objetos y Tecnologías de Aprendizaje. Congreso llevado a cabo en Manizales, Colombia.

Pérez-Then, E. (2020). Nuevo coronavirus 2019-ncov: impacto en salud global. Ciencia y Salud, 4(1), 5-9.

Porras, A. A., Castilla, I., \& Rivera, K. (2017). e-Learning: Rompiendo fronteras. Redes de Ingenierâia, 91-100. https://doi.org/10.14483/2248762X.12480

Rincón, L. M., \& Castilla, C. A. (2018). Flipped Classroom: Aula Invertida en la Formación de Psicólogos. CINA RESEARCH, 2(2), 17-23.

Sangrà, A. (2020). Decálogo para estudiar en línea en tiempos de coronavirus. Recuperado de: https://www.uoc.edu/portal/es/news/actualitat/2020/167decalogo-estudiar-linea.html

Talbert, R. (2012). Inverted classroom. Colleagues, 9(1), Article 7. Recuperado http://scholarworks.gvsu.edu/colleagues/vol9/iss1/7

Thamhain, H. J. (2005). Management of technology: Managing effectively in technology-intensive organizations. John Wiley \& Sons.

Unesco. (2020). COVID-19 Interrupción educativa y respuesta. Recuperado de: https://en.unesco.org/covid19/educationresponse 